\title{
UNIFORM CONVERGENCE FOR A HYPERSPACE
}

\author{
R. J. GAZIK
}

\begin{abstract}
In this note a uniform convergence in the collection $C(E)$ of nonempty, compact subsets of a separated uniform convergence space $E$ is defined. This convergence is compared with the hyperspace convergence on $C(E)$ and it is shown that the two convergences agree on Richardson's class $\Gamma$. In the case of a regular $T_{1}$ topological space $(E, t)$ this means that there is a uniform convergence structure on $E$, which induces $t$, such that uniform convergence in $C(E)$ is convergences in the Vietoris topology on $C(E)$.
\end{abstract}

1. Definition of uniform convergence. Let $C(E)$ be the collection of nonempty, compact subsets of a Hausdorff topological space $(E, t)$. Then $C(E)$ may be equipped with the Vietoris topology. If, in addition, $(E, t)$ is completely regular and $\mathscr{U}$ is a uniform structure which induces $t$, then $C(E)$ also carries the uniform topology. A classical result is that the Vietoris and uniform topologies agree on $C(E)$ (see [6]).

Now let $C(E)$ be the collection of nonempty, compact subsets of a Hausdorff convergence space $(E, t)$ (see [3]). There is a reasonable way to generalize the Vietoris and uniform topologies to this setting. In fact, in [4] a convergence $h(t)$ for $C(E)$ was defined. It was shown that $h(t)$ is the Vietoris topology for topological $t$. Moreover $h(t)$ - is Hausdorff (regular) (compact) whenever $t$ is Hausdorff (regular) (compact and regular). But no additional properties of $t$ (such as complete regularity) are needed to make a reasonable definition of uniform convergence in $C(E)$ and this is done below.

Let $(E, \mathscr{J})$ be a separated uniform convergence space (see [2]). In [1] Cochran defined a $U^{*}$ base for $\mathscr{J}$ to be a base $\beta$ for $\mathscr{J}$ such that each member of $\beta$ is coarser than the diagonal filter, each member of $\beta$ is its own inverse, the composition of any two members of $\beta$ exists and is finer than a third member, and the infimum of two members of $\beta$ is again in $\beta$. Each uniform convergence space has a $U^{*}$ base; put $\beta=(J \in \mathscr{J}: J \leqq$ $\left.[\Delta], J=J^{-1}\right)$. It should also be pointed out that each Hausdorff topological space $(E, t)$, indeed each Hausdorff convergence space $(E, t)$, has a uniform convergence structure $\mathscr{J}$, constructively defined, such that

Received by the editors February 5, 1973.

AMS (MOS) subject classifications (1970). Primary 54A05, 54A20, 54B20, 54E15.

Key words and phrases. Vietoris topology, uniform topology, convergence spaces, uniform convergence spaces, hyperspaces of convergence spaces.

(c) American Mathematical Society 1974 
$t(\mathscr{J})$-the convergence induced by $\mathscr{J}$-is the same as $t$ (Cochran [1] and Keller [5]).

REMARK. With respect to the definition below, notice that the convergence defined depends on $\mathscr{J}$ but not on the choice of a $U^{*}$ base for $\mathscr{J}$.

Definition. Let $C(E)$ be the collection of nonempty, $t(\mathscr{J})$ compact subsets of a separated uniform convergence space $(E, \mathscr{J})$ and let $\beta$ be a $U^{*}$ base for $\mathscr{J}$. For $J \in \beta, V \in J$, define $T(J, V)$ to be the set of all ordered pairs $(A, B), A, B \in C(E)$, such that $A \subset V(B), B \subset V(A)$, and define $T(J)$ to be the filter generated by the $T(J, V), V \in J$. The structure $\mathscr{J}$ of uniform convergence in $C(E)$ is the uniform convergence structure generated by the $T(J), J \in \beta$.

THEOREM 1. Let $(E, \mathscr{J})$ be a separated uniform convergence space. Then

(a) $\mathscr{J}$ indeed is a uniform convergence structure for $C(E)$;

(b) if $\mathscr{J}$ is a uniform structure then $t(\mathscr{J})$ is the uniform topology;

(c) a filter $\Phi$ on $C(E)$ converges relative to $t(\mathscr{J})$ to $A \in C(E)$ if and only if $\Phi \times \dot{A} \geqq T(J)$ for some $J \in \beta$.

Proof. (a) Since $\beta$ is a $U^{*}$ base, each member of $\beta$ contains the diagonal so $T(J, V) \neq \varnothing$ and $T(J)$ is a filter. If $D$ is the diagonal filter in $C(E) \times$ $C(E)$ then $D \geqq T([\Delta])$ so $D \in \mathscr{J}$. Each member of $\beta$ is its own inverse, hence $(T(J))^{-1} \geqq T(J)$. Since each member of $\mathscr{J}$ is finer than a finite infimum of $T(J)$ 's, it follows that the inverse of a member of $\mathscr{J}$ is again in $\mathscr{J}$. Finally, notice that if $J, J_{1}, J_{2}, \cdots, J_{n} \in \beta$ then

(1) $T(J) \circ\left(\bigwedge T\left(J_{i}\right)\right) \geqq \bigwedge T\left(J \circ J_{i} \wedge J_{i} \circ J\right)$. Since each $J \circ J_{i} \wedge J_{i} \circ J$ is finer than a member of $\beta$ we may apply (1) repeatedly to show that the composition of two members of $\mathscr{J}$ is again in $\mathscr{J}$ when it exists.

(b) If $\mathscr{J}$ is a uniform structure, $\mathscr{J}$ itself is a $U^{*}$ base of one element and it is obvious that $t(\mathscr{J})$ is the uniform topology.

(c) This follows from the definition, the fact that $\beta$ is a $U^{*}$ base and the inequality $\bigwedge T\left(J_{i}\right) \geqq T\left(\bigwedge J_{i}\right)$.

2. Comparison of $t(\mathscr{J})$ and $h(t(\mathscr{J}))$. If $C(E)$ is the collection of nonempty $t(\mathscr{J})$ compact subsets of a separated uniform convergence space $(E, \mathscr{J})$, there are two convergences defined on $C(E)$, namely the convergence $t(\mathscr{J})$ defined in $\S 1$ and the hyperspace convergence $h(t(\mathscr{J}))$ of [4] with respect to the convergence $t(\mathscr{J})$ induced by $\mathscr{J}$. Theorem 2 below compares these in general.

Notice that a filter $\alpha$ in a uniform convergence space $(E, \mathscr{J}) t(\mathscr{J})$ accumulates at $x \in E$ if and only if there exists $J \in \mathscr{J}$, such that $F \in \alpha$ and $V \in J$ implies $F \cap V(x) \neq \varnothing$. We abbreviate this by saying " $\alpha t(\mathscr{J})$ accumulates at $x$ with respect to $J$ ". 
THEOREM 2. If $(E, \mathscr{J})$ is a separated uniform convergence space, $t(\mathscr{J}) \geqq h(t(\mathscr{J}))$.

Proof. Let $\Phi \rightarrow A$ relative to $t(\mathscr{J})$ so that $\Phi \times \dot{A} \geqq T(J)$ for some $J \in \beta, \beta$ a $U^{*}$ base for $\mathscr{I}$. In order to show that $\Phi h(t(\mathscr{J}))$ converges to $A$ it must be proved that (1) and (2) of Definition 2.1 of [4] hold.

Proof of (1). Suppose that $(x, g)$ is a selection of a cofinal segment $(D, f)$ of $\Phi$. Let $V \in J$ and $d \in D$.

(a) There exists $r(V) \in \Phi$ such that $B=V^{\prime}(A)$ for each $B \in r(V)$.

(b) There exists $p(d, V) \geqq d$ such that $f(p(d, V)) \subset r(V)$. The statements (a) and (b) ceme, respectively, from the convergence of $\Phi$ and the fact that $(D, f)$ is a cofinal segment of $\Phi$. Since $(x, g)$ is a selection of $(D, f)$, (a) and (b) imply

(c) $(x(p(d, V)), a(p(d, V))) \in V$ for some $a(p(d, V)) \in A$. Direct $D \times J$ with the product order; that is $(d, V) \geqq(e, U)$ if and only if $d \geqq e$ and $V \subset U$. Then $(a(p(d, V)):(d, V) \in D \times J)$ is a net in $A$ whose section filter accumulates at some $a \in A$ with respect to some $K \in \beta$. ( $A$ is compact.)

Now let $V \in J, W \in K, d \in D$. From the remarks above there is an $(e, U) \geqq(d, V)$ such that $(a(p(e, U)), a) \in W$. By (c)

$$
(x(p(e, U)), a(p(e, U))) \in U \subset V .
$$

These last two statements mean that $(x(p(e, U)), a) \in V \circ W$ with $p(e, U) \geqq d$. This in turn means that the section filter $S(x) t(\mathscr{J})$ accumulates at $a$ with respect to $J \circ K \in \mathscr{J}$. Hence $S(x) t(\mathscr{J})$ accumulates at a point of $A$.

Proof of (2). Let $a \in A$. If $(V, r) \in J \times \Phi$ use the convergence of $\Phi$ to see that there is an $f(V, r) \subset r, f(V, r) \in \Phi$, such that $A \subset V(B)$ whenever $B \in f(V, r)$. With $J \times \Phi$ ordered with the product order, $(J \times \Phi, f)$ is a cofinal segment of $\Phi$. Also, clearly, $J(a) \rightarrow a$. It is asserted that $(J \times \Phi, f)$ and $J(a)$ satisfy (2) of Definition 2.1 of [4]. For, if $V(a) \in J(a)$ and $r \in \Phi$ there is a $U \in J$ such that $U^{-1} \subset V .\left(J=J^{-1}\right.$ for $J \in \beta$.) Whenever $B \in f(W, s)$, $(W, s) \geqq(U, r)$, the relation $A \subset W(B) \subset U(B)$ is a consequence of the properties of $f$ so $(a, b) \in U$ for some $b \in B$. Then $(b, a) \in U^{-1} \subset V$, hence $B \cap V(a) \neq \varnothing$. This completes the proof.

Without restrictions on $\mathscr{J}$ we cannot hope to obtain equality in Theorem 2 , even if very strong conditions are placed on $t(\mathscr{J})$. The following illustrates the point.

ExAmple. Let $(E, t)$ be a Hausdorff topological space and let $S$ be a finite subset of $E$. Define $T(S)=\wedge(N(x) \times N(x): x \in S) \wedge D$ where $N(x)$ is the neighborhood filter at $x$ and $D$ is the diagonal filter. It is easy to see that the collection of $T(S), S$ finite, generates a uniform convergence structure $\mathscr{F}$ on $E$ and, in fact, $t(\mathscr{F})=t$. 
In particular, let $(E, t)$ be the closed unit disk with the usual topology. By Theorem 2.2 of [4], $h(t(\mathscr{J}))$ is the Vietoris topology on $C(E)$. But notice that $t(\mathscr{J})$ is not compact, even though $(E, \mathscr{J})$ is. Hence uniform convergence in $C(E)$ is not the same as the hyperspace convergence $h(t(\mathscr{J}))$.

With respect to this example and Theorem 3.7 of [4] one might reasonably ask the following question. If $(E, \mathscr{J})$ is compact and regular, does there exist a uniform convergence structure $\mathscr{K}$ for $E$, with $t(\mathscr{K})=t(\mathscr{J})$, such that $t(\mathscr{K})$ is compact? The author does not know the answer.

Richardson [7] defined a class $\Gamma$ of uniform convergence structures for $E$ as follows: $\mathscr{J} \in \Gamma$ if and only if there exists $J \in \mathscr{J}$ such that whenever $x \in E$ and $\alpha \rightarrow x$, then $\alpha \geqq J(x)$. We will call such a $J$ a fixed member of $\mathscr{J}$ for convenience. A fixed member of $\mathscr{J}$ may be taken to be symmetric and coarser than the diagonal filter.

LemMa 1. Let $\mathscr{J} \in \Gamma$ with $J$ a fixed member of $\mathscr{J}$. If $A$ is a compact subset of $(E, \mathscr{J})$ and $V \in J$, then $A \subset V(F)$ for some finite subset $F$ of $A$.

Proof. If not, $A \cap(V(F))^{\prime} \neq \varnothing$ for each finite subset $F$ of $A$. The filter generated by the $A \cap(V(F))^{\prime}$ accumulates at some point $a \in A$ with respect to the same $J$. Then $A \cap(V(a))^{\prime} \cap V(a) \neq \varnothing-$ a contradiction.

THEOREM 3. If $(E, \mathscr{J})$ is separated and $\mathscr{J} \in \Gamma$ then $t(\mathscr{J})=h(t(\mathscr{J}))$.

Proof. Let $\beta$ be a $U^{*}$ base for $\mathscr{J}$ and suppose $\Phi \rightarrow A$ relative to $h(t(\mathscr{J}))$. In order to show that $\Phi \rightarrow A$ relative to $t(\mathscr{J})$ it suffices to prove the following.

(a) For some $K \in \mathscr{J}$, whenever $U \in K$ there exists $r \in \Phi$ such that $B \subset$ $U(A)$ for all $B \in r$.

(b) For some $L \in \mathscr{J}$, whenever $U \in L$ there exists $r \in \Phi$ such that $A \subset U(B)$ for all $B \in r$.

Proof of (a). Suppose (a) is false for $K=J, J$ a fixed member of $\mathscr{J}$. There is some $V \in J$ such that, whenever $r \in \Phi, g(r) \notin V(A)$ for some $g(r) \in r$. Hence there exists $x(r) \in g(r)-V(A)$. If $\Phi$ is ordered by $r \geqq s$ if $r \subset s$, and $f$ is the identity map on $\Phi$, then $(x, g)$ is a selection of the cofinal segment $(\Phi, f)$ of $\Phi$. Since $\Phi \rightarrow A$ relative to $h(t(\mathscr{J})), S(x)$-the section filter of $x$-accumulates at some $a \in A$ with respect to $J$ (for $\mathscr{J} \in \Gamma$ ). Thus, if $r$ is arbitrary in $\Phi$ there is an $s \subset r$ for which $x(s) \in V(a)$. But this contradicts $x(s) \notin V(A)$.

Proof of (b). We assert that (b) is true for $L=J \circ J, J$ a fixed member of $\mathscr{J}$. By Lemma 1:

(1) If $V \in J, A \subset V(F)$ for some finite subset $F \subset A$. The following is also true. 
(2) If $a \in A, V \in J$, there exists $r(a, V) \in \Phi$ such that whenever $B \in r(a, V)$, then $a \in V(B)$.

For suppose (2) is false. For $r \in \Phi$ there exists $g(r) \in r$ with $a \notin V(g(r))$. The filter $\alpha$ of Definition 2.1 of [4] is finer than $J(a)$ so that, for some $r$, $g(r) \cap U(a) \neq \varnothing, U^{-1} \subset V, U \in J$. From this, there is some $y \in g(r)$ such that $a \in U^{-1}(y) \subset V(y) \subset V(g(r))$. This is a contradiction and (2) holds.

Finally, let $V \circ V \in J \circ J$. Use (1) and (2) and put $r=\bigcap(r(a, V): a \in F)$. Let $B \in r$ and $z \in A$. Then $z \in V(a)$ for some $a \in F$ and $a \in V(B)$ so $z \in$ $(V \circ V)(B)$ follows and we conclude that $A \subset(V \circ V)(B)$. This completes the proof.

Theorem 4 is an immediate consequence of the result above, Theorem 2.2 of [4], and the major result of [7]. In this regard notice that in [7] Richardson actually constructs $\mathscr{J}$ from the neighborhood filters, so that $\mathscr{J}$ is constructively defined.

THEOREM 4. Let $C(E)$ be the collection of nonempty, compact subsets of a regular $T_{1}$ topological space $(E, t)$. There exists a uniform convergence structure $\mathscr{J}$ for $E$, with $t(\mathscr{J})=t$, such that $t(\mathscr{J})$ is the Vietoris topology on $C(E)$.

Necessary and sufficient conditions on $\mathscr{J}$ which insure $t(\mathscr{J})=h(t(\mathscr{J}))$ would be very interesting. The author knows only the partial result in Theorem 3.

\section{REFERENCES}

1. A. C. Cochran, On uniform convergence structures and convergence spaces, Doctoral Dissertation, University of Oklahoma, Norman, Okla., 1966.

2. C. H. Cook and H. R. Fischer, Uniform convergence structures, Math. Ann. 173 (1967), 290-306. MR 36 \#845.

3. H. R. Fischer, Limesräume, Math. Ann. 137 (1959), 269-303. MR 22 \#225.

4. R. J. Gazik, A hyperspace for convergence spaces, Proc. Amer. Math. Soc. 37 (1973), 234-240.

5. H. H. Keller, Die Uniformiserbarkeit des Limesräumes, Math. Ann. 176 (1968), 334-341. MR 37 \#874.

6. E. A. Michael, Topologies on spaces of subsets, Trans. Amer. Math. Soc. 71 (1951), 152-182. MR 13, 54.

7. G. D. Richardson, A class of uniform convergence structures, Proc. Amer. Math. Soc. 25 (1970), 399-402. MR 41 \#991.

Department of Mathematics, Arkansas State University, State University, ARKansas 72467

Current address: Department of Mathematics, Washington State University, Pullman, Washington 99163 